\title{
Virtual psychiatric care fast-tracked: reflections inspired by the COVID-19 pandemic
}

\author{
Tea Rosic, ${ }^{1} \odot$ Sandra Lubert, ${ }^{2}$ Zainab Samaan ${ }^{1}$ (
}

BJPsych Bulletin (2022) 46, 33-36, doi:10.1192/bjb.2020.97

'McMaster University, Hamilton, Ontario, Canada; ${ }^{2}$ Hamilton, Ontario, Canada

Correspondence to Zainab Samaan (samaanz@mcmaster.ca)

First received 7 Jun 2020, final revision 19 Jul 2020, accepted 12 Aug 2020

(C) The Author(s), 2020. Published by Cambridge University Press on behalf of BJPsych Bulletin. This is an Open Access article, distributed under the terms of the Creative Commons Attribution licence (http://creativecommons.org/ licenses/by/4.0/), which permits unrestricted re-use, distribution, and reproduction in any medium, provided the original work is properly cited.
Summary For many patients and healthcare providers, the move to virtual psychiatric care has been fast-tracked by the COVID-19 pandemic. In this article, we consider a patient perspective and a provider perspective on the transition to virtual psychiatric care and its strengths and limitations, as well as a call for much-needed future research.

Keywords Telemedicine; telepsychiatry; virtual care; COVID-19; pandemic.
The COVID-19 pandemic has rapidly transformed service delivery, including the delivery of medical care, around the world. As jurisdictions worldwide enacted measures to slow the spread of the SARS-CoV-2 virus, service providers quickly adapted. What was previously a slow expansion of telemedicine and virtual care over the past two decades, with the primary goal of overcoming geographical barriers, ${ }^{1}$ became suddenly fast-tracked in a matter of weeks. A rapid transition to virtual psychiatric care, care provided by telephone or video, is one such transformation resulting from the COVID-19 pandemic and the measures implemented to contain it.

Leaders and advocates in the field of telepsychiatry are expecting, and in fact encouraging, these changes to persist post-pandemic..$^{2-4}$ The aim of this article is to reflect on the transition to virtual psychiatric care through three lenses: a patient perspective, a provider perspective and a scholarly perspective, including a call for future research. The patient and provider perspectives obtained for this article reflect individual experiences with the transition to virtual psychiatric care and are not intended to capture the diversity of perspectives and experiences that may exist. The patient and providers have experienced both models of care pre- and post-pandemic onset.

\section{A patient perspective, by Ms Sandra Lubert}

'I am a mental health patient with a diagnosis of depression, PTSD and chronic sleep disruption. I have been either ill or in remission for many years and have experienced extensive clinical intervention both before and during the COVID-19 pandemic; that is, both actual, in-person care and virtual, online care.
Clearly, a physical meeting between patient and practitioner in a clinical environment is often warranted. Face-to-actualface is sometimes best, and I am not advocating the replacement of such visits with online care. Further, some of our most vulnerable populations lack access to telehealth and/ or virtual technology and for these individuals, it is imperative that we either provide them with the tools required to access proposed alternative care or continue to provide traditional, in-person interventions. Barriers to online care can be significant, salient features and these need to be considered. When a physical appointment is required or even simply preferred, this must be accommodated. I believe that an ideal system of mental health care features a robust, multifaceted approach which meets the needs of all patients and practitioners.

My own mental illness includes severe insomnia and related suicidality. Six weeks ago - right around the onset of the pandemic - I had a psychiatric crisis requiring an ER visit and overnight hospitalization. This urgent, on-site care was life-saving. But I believe that my follow-up care, which has been entirely virtual, has been just as critical. This recent experience leads me to believe that the rapid changes necessitated by COVID-19 have a great deal to teach us about psychiatric care delivery.

For me, as a recipient, 'pandemic-period psychiatry' (via eVisits) has not only been adequate and helpful, it has in many ways been far more effective than its in-person counterpart. There are three main aspects of online care that have enhanced the overall experience for me: reduced cost, ease of access, and increased flexibility/comfort. From a very practical standpoint, I can state that eVisits have saved me money. I have not had to pay for gas to get to and from appointments, and I have not had to pay for parking. Many patients travel a significant distance in order to see clinicians, and this can be very expensive. Some rely on caregivers, taxis, or public transit. Again, while this may seem insignificant, 
the cost of transportation can be a major deterrent in seeking treatment, particularly for those on a limited income.

Ease of access around virtual care is a huge factor. My mental illness is often incapacitating; when I am in crisis, it is difficult for me to get out of bed, let alone leave my house. Things like getting dressed, planning for travel time, being presentable may seem trivial, but for those struggling with severe mental illness, these obstacles can be insurmountable. The anxiety induced by having to leave the house and get to one's doctor is sometimes crippling and prevents us from seeking care. When I am in distress, the idea of sitting in a waiting room (often in tears, usually in psychic pain) is intolerable. For those dealing with a physical disability in addition to mental illness, barriers are even more debilitating. When access is restricted, the situation with mental health can become dire. Indeed, it can even become deadly. eVisits have saved me; I have been better able to access consistent, necessary psychiatric care because I can do so virtually from my home.

Increased flexibility is perhaps the most beneficial aspect of virtual care. Since the variables of transportation and location are removed, both patient and practitioner are better able to schedule meetings. Modifying appointments has also been far easier to accomplish online, with recipient and caregiver both having immediate access to electronic calendars and other tools. Involving my partner in an eVisit is merely a matter of inviting her to sit next to me at my computer. Both my doctor and I have information at our fingertips if needed.

Finally, I feel more comfortable with eVisits. Meeting with my practitioner from my own environment puts me more at ease. Ultimately, this has to do with a levelling effect - it makes me feel like I am more of an equal in the patient-practitioner exchange. Traditional appointments take place in the doctor's realm - their office, clinic, the hospital... in some ways, this gives them the 'home field advantage', as it were. I am in their space. They know where everything is, who everyone is, but it is largely unfamiliar to me. I am a guest. I don't have my own mug, for tea.'

\section{A provider perspective, by Dr Tea Rosic, psychiatry resident, and Dr Zainab Samaan, staff psychiatrist}

'Suddenly, going to the clinic to see patients is a health risk to both my patients and me. I am faced with a precarious balancing act as I find ways to provide adequate psychiatric care to patients who face increasing anxiety, depression, social isolation, and changes in mental status during the pandemic. The novelty of COVID-19, the ever-dynamic guidelines, processes, and instructions, issues of shortage and conservation of PPE, and new risk-benefit equilibria as we consider each clinical decision are all present in ways we have not encountered before.

Virtual care has alleviated the risk carried by face-to-face contact but has raised many new challenges. Do I have a printer, fax machine, dedicated telephone line, secure email, and contact information for community pharmacies? Do I know how to schedule appointments without administrative staff support? New medicolegal challenges arise: what if a patient has an urgent psychiatric need, should I be available online $24 \mathrm{~h} /$ day? Can we send patients to hospital or are we contributing to risk of transmission and placing patients at greater risk? Not unlike before the pandemic, my patients in greatest need of care often face the greatest barriers in accessing it: limited mobile phone minutes, barriers in access to internet and virtual technologies, lack of privacy.

Nevertheless, virtual care has been transformative. Being able to provide much needed care despite the pandemic restrictions is satisfying. Seeing patients through telemedicine modalities has opened the door to better assessments of their environment and allows for easier involvement of other family members when invited. I see patients' pets and other important aspects of their lives. How often does it occur, in the clinic, that we have no access to updated medication lists, and how much time is spent trying to gain this information before making further treatment recommendations? With virtual visits and medications accessible to the patient in their home, this problem is averted.

Virtual care during this pandemic has so quickly transformed how (and from where) we do our jobs. I have greater control over my schedule, working hours are more flexible and I am more available outside of structured clinical time. How these changes will evolve following the pandemic is unknown, but there is much to be learned and gained from this experience.'

\section{A scholarly perspective: the evidence base}

Virtual psychiatric care has an established evidence base and has shown effectiveness in a variety of areas, including within different patient populations and in different clinical settings. ${ }^{5}$ Previous research suggests that building therapeutic rapport is just as effective virtually as it is in person. ${ }^{5}$ For clinicians thrust into providing virtual psychiatric care, the strengths and limitations of this model of service delivery are becoming clearer. Recognising and reflecting on these is just as important now as it will be post-pandemic, when organisations and providers decide on the models of care they will offer. In the post-pandemic era, in-person healthcare may indeed become 'option B' for many patients. ${ }^{6}$ We are working through the technical issues, concerns about confidentiality and provider payment obstacles that each slowed the advance of virtual care in the past. For many patients, as described above, virtual care provides the easier access, flexibility and comfort that is lacking from in-person, hospital- or clinic-based care. Some patients may be greatly benefited by ongoing virtual appointments.

For all of the potential benefits of virtual psychiatric care, there are shortcomings that must be considered. Physical examination cannot be conducted as usual and there may be challenges in comprehensively assessing physical appearance and functioning in virtual psychiatric assessments. Virtual care may impose additional barriers to assessment for patients presenting with certain symptoms, such as paranoid ideation, ${ }^{7,8}$ although a recent study on the use of telepsychiatry for first-episode psychosis suggests that $50 \%$ of patients reported telepsychiatry as a favourable modality for follow-up., ${ }^{9,10}$ Patients experience differential access to virtual platforms and technology, based on socioeconomic and other factors. ${ }^{11}$ In particular, patients with severe and persistent mental illness may face even greater barriers to accessing care virtually than the general patient population. ${ }^{8}$ Individuals living in close quarters with multiple family members may have insufficient space to talk while maintaining privacy and confidentiality. ${ }^{11,12}$ Older adults and individuals with disabilities may face challenges with access to technology, visual impairment or hearing impairment, creating barriers and gaps in care. Cultural factors in virtual care must be carefully considered and addressed. Patients who require language interpreters may be disadvantaged by virtual psychiatric care. ${ }^{13}$ With the expansion of virtual psychiatric care, we must be particularly mindful of the risk of widening the gap in access to care for patients who are marginalised or otherwise vulnerable. The broader issue of global healthcare access inequities in telepsychiatry is being raised. ${ }^{14}$ 


\section{Research and practice implications}

In this article, we aimed to provide both patient and provider perspectives on the rapid and monumental shift to virtual psychiatric care that occurred, seemingly overnight, in many jurisdictions. However, the perspectives shared may be limited in their generalisability for settings outside of a well-funded public healthcare system or in places with limited existing capacity for the provision of telemedicine. We stress that the experiences of unique patients and unique providers, working in diverse clinical settings, managing different clinical presentations and operating in distinct healthcare systems worldwide might be critically different. The global healthcare community will benefit from hearing and learning from diverse experiences and perspectives.

As we rapidly usher in this new era of virtual psychiatric care, concerted efforts must be made to study and learn from our experiences. Research must be undertaken to examine the impact of these changes in psychiatric service delivery for different patient groups and different providers. Ensuring identification of individuals and groups whose needs are not met will be critical. Qualitative research that can capture the depth and detail of our human experiences with virtual psychiatric care will be necessary. Economic analyses of the costs and savings of this model will also be integral. There is some pre-pandemic evidence to suggest great potential cost savings with widespread use of telemedicine - including savings accrued from shorter time spent travelling and waiting, for both patients and providers. ${ }^{15,16}$

Clinical and research groups worldwide have begun to publish and share their experiences in implementing telepsychiatry during the COVID-19 pandemic for patient populations in various settings, including child and adolescent psychiatry, ${ }^{17}$ general out-patient psychiatry ${ }^{11,13}$ and in-patient psychiatry. ${ }^{18}$ Surveys of psychiatrists using telepsychiatry during the pandemic indicate benefits such as convenience and flexibility, as well as challenges in relation to the use of technology, impact on confidence in diagnosis and impact on therapeutic alliance. ${ }^{13}$ Authors are giving consideration to the experience of trainees and educators using telepsychiatry. ${ }^{19}$

The COVID-19 pandemic propelled us into a new era of virtual psychiatric care, and opened the door to a re-evaluation of how, and why, we provide mental healthcare in the ways we do. This door will remain open, post-pandemic, allowing us to rigorously evaluate, shape and refine our models of care to meet the needs of our patients as best as possible.

\section{About the authors}

Tea Rosic, MD, is a Resident Physician in the Department of Psychiatry and Behavioural Neurosciences and a PhD student in the Department of Health, Evidence and Impact at McMaster University, Hamilton, Ontario, Canada. Sandra Lubert, BA, BEd, is a writer/author and educational consultant in Hamilton, Ontario, Canada. Zainab Samaan, MBChB, MSc, DMMD, MRCPsych, PhD, is Associate Professor of Psychiatry and Behavioural Neurosciences in the Department of Health Research Methods, Evidence, and Impact at McMaster University, and Director of the Clinician Investigator Program within the Mood Disorders Program, St Joseph's Healthcare, Hamilton, Ontario, Canada.

\section{Author contributions}

All three authors are responsible for the article concept and design. T.R. conducted the literature search. All three authors contributed to writing the first draft of the manuscript and critically revising the final manuscript; all three reviewed and approved the final manuscript.

\section{Declaration of interest}

Z.S's research is supported by the Canadian Institutes of Health Research (CIHR).

ICMJE forms are in the supplementary material, available online at https:// doi.org/10.1192/bjb.2020.97

\section{References}

1 Bashshur RL, Shannon GW. History of Telemedicine: Evolution, Context, and Transformation. Mary Ann Liebert, 2009: 384-90.

2 Shore JH, Schneck CD, Mishkind MC. Telepsychiatry and the coronavirus disease 2019 pandemic: current and future outcomes of the rapid virtualization of psychiatric care. JAMA Psychiatry [Epub ahead of print] 11 May 2020. Available from: https://doi.org/10.1001/jamapsychiatry.2020.1643.

3 Torous J, Jän Myrick K, Rauseo-Ricupero N, Firth J. Digital mental health and COVID-19: using technology today to accelerate the curve on access and quality tomorrow. JMIR Ment Health 2020; 7(3): e18848.

4 Smith K, Ostinelli E, Cipriani A. COVID-19 and mental health: a transformational opportunity to apply an evidence-based approach to clinical practice and research. Evid Based Ment Health 2020; 23: 45-6.

5 Hilty DM, Ferrer DC, Parish MB, Johnston B, Callahan EJ, Yellowlees PM. The effectiveness of telemental health: a 2013 review. Telemed J E Health 2013; 19: 444-54.

6 Duffy S, Lee TH. In-person health care as option B. N Engl J Med 2018; 378: $104-6$

7 Wong KTG, Liu D, Balzan R, King D, Galletly C. Smartphone and internet access and utilization by people with schizophrenia in South Australia: quantitative survey study. JMIR Ment Health 2020; 7(1): e11551.

8 Zulfic Z, Liu D, Lloyd C, Rowan J, Schubert KO. Is telepsychiatry care a realistic option for community mental health services during the COVID-19 pandemic? Aust N Z J Psychiatry [Epub ahead of print] 22 Jun 2020. Available from: https://doi.org/10.1177/0004867420937788.

9 Lal S, Abdel-Baki A, Sujanani S, Bourbeau F, Sahed I, Whitehead J. Perspectives of young adults on receiving telepsychiatry services in an urban early intervention program for first-episode psychosis: a cross-sectional, descriptive survey study. Front Psychiatry 2020; 11: 117.

10 Santesteban-Echarri O, Piskulic D, Nyman RK, Addington J. Telehealth interventions for schizophrenia-spectrum disorders and clinical highrisk for psychosis individuals: a scoping review. J Telemed Telecare 2020; 26: 14-20.

11 Yellowlees P, Nakagawa K, Pakyurek M, Hanson A, Elder J, Kales HC. Rapid conversion of an outpatient psychiatric clinic to a $100 \%$ virtual telepsychiatry clinic in response to COVID-19. Psychiatr Serv 2020; 71: 749-52.

12 Chin HP, Palchik G. Telepsychiatry in the age of COVID: some ethical considerations. Camb Q Healthc Ethics [Epub ahead of print] 24 Jun 2020. Available from: https://doi.org/10.1017/S0963180120000523.

13 Olwill C, McNally D, Douglas L. Psychiatrist experience of remote consultations by telephone in an outpatient psychiatric department during the COVID-19 pandemic. Ir J Psychol Med [Epub ahead of print] 22 May 2020. Available from: https://doi.org/10.1017/ipm. 2020.51.

14 Ramalho R, Adiukwu F, Bytyci DG, El Hayek S, Gonzalez-Diaz JM Larnaout $A$, et al. Telepsychiatry and healthcare access inequities during the COVID-19 pandemic. Asian J Psychiatr [Epub ahead of print] 16 Jun 2020. Available from: https://doi.org/10.1016/j.ajp.2020.102234

15 Canada Health Infoway. Telehealth Benefits and Adoption: Connecting People and Providers Across Canada. Canada Health Infoway, 2011.

16 Conference Board of Canada. Valuing Time Saved: Assessing the Impact of Patient Time Saved from the Adoption of Consumer Health Solutions. Canada Health Infoway, 2012

17 Sharma A, Sasser T, Schoenfelder Gonzalez E, Vander Stoep A, Myers $\mathrm{K}$. Implementation of home-based telemental health in a large child 
psychiatry department during the COVID-19 crisis. J Child Adolesc Psychopharmacol [Epub ahead of print] 8 Jul 2020. Available from: https://doi.org/10.1089/cap.2020.0062.

18 Kalin ML, Garlow SJ, Thertus K, Peterson MJ. Rapid implementation of telehealth in hospital psychiatry in response to COVID-19. Am J Psychiatry 2020; 177: 636-7.
19 Richards M, DeBonis K. Psychiatric training during a global pandemic: how COVID-19 has affected clinical care, teaching, and trainee wellbeing. Psychiatr Serv [Epub ahead of print] 30 Jun 2020. Available from: https://doi.org/10.1176/appi.ps.202000277.

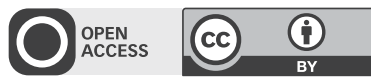

\title{
SPECIAL ARTICLE
}

\section{The contagiousness of memes: containing the spread of COVID-19 conspiracy theories in a forensic psychiatric hospital}

\author{
Reena Panchal, ${ }^{1}$ (1) Alexander Jack ${ }^{2}$ (1)
}

BJPsych Bulletin (2022) 46, 36-42, doi:10.1192/bjb.2020.120

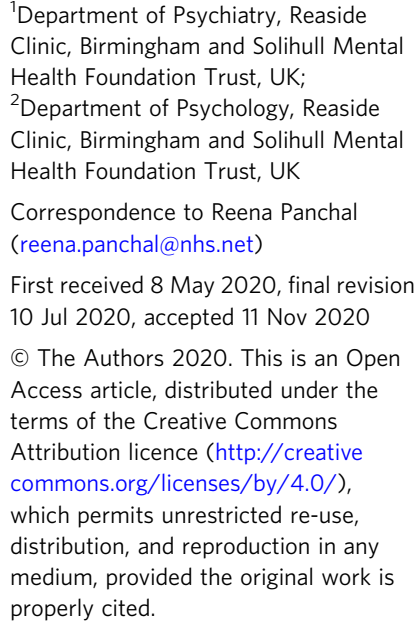

'Department of Psychiatry, Reaside Clinic, Birmingham and Solihull Mental Health Foundation Trust, UK

${ }^{2}$ Department of Psychology, Reaside

Clinic, Birmingham and Solihull Mental Health Foundation Trust, UK

Correspondence to Reena Panchal (reena.panchal@nhs.net)

First received 8 May 2020, final revision 10 Jul 2020, accepted 11 Nov 2020 (c) The Authors 2020. This is an Open Access article, distributed under the terms of the Creative Commons Attribution licence (http://creative commons.org/licenses/by/4.0/), which permits unrestricted re-use, distribution, and reproduction in any medium, provided the original work is properly cited.

Summary COVID-19 has transformed healthcare service provision. In addition to the spread of a virus, there has been an equally concerning emergence and spread of conspiracy theories. Such theories can threaten societal cohesion and adherence to the necessary public health guidance. In a forensic in-patient setting, such difficulties can be amplified. In this paper, we outline the key theory in relation to the development and spread of conspiracy theory memes. We propose primary, secondary and tertiary level responses to tackle the possible generation and spread of harmful conspiracies in the forensic in-patient setting. We consider this to be important, as there is a risk that such beliefs could affect patients' mental health and, in extremis, undermine physical health efforts to reduce the spread of COVID-19.

Keywords COVID-19; forensic mental health services; conspiracy theory; meme; multidisciplinary working.
Forty-four cases of pneumonia of unknown microbial origin were reported to the World Health Organization (WHO) on 31 December 2019. ${ }^{1}$ Investigations revealed that the culprit organism was a novel coronavirus, dubbed COVID-19. COVID-19 has spread quicker than experts anticipated; the WHO declared an international state of emergency - a true pandemic - in early March 2020, as the virus spread rapidly between continents. The human cost has been, and continues to be, vast.

\section{The public health response}

The global response to COVID-19 has emphasised the necessity for reduced close contact; hence, the intervention termed 'social distancing'. To achieve this aim, many governments implemented 'lockdown' strategies to limit the free movement of the public, although the precise restrictions and severity of the measures have differed from country to country. The UK government urged people to 'Stay
Home, Save Lives, Protect the NHS', with only essential travel permitted, restricted mixing of households and citizens at one point limited to a single exercise outing per day. There was a national drive to 'flatten the curve', with the stated intention to avoid overwhelming the National Health Service (NHS). A further patriotic message resonated with the public; that is, to protect the most vulnerable in society. Ultimately, the effectiveness of government and society's efforts to maintain this unconventional and rather antisocial injunction will be measured by the number of casualties.

\section{A forensic mental health hospital facing the pandemic}

We are based in a psychiatric medium secure unit (MSU) in the West Midlands, UK. The MSU has capacity for 90 male patients across multiple wards and provides care to men who present with complex risk behaviours and experience 